\title{
Intravitreal Ranibizumab Combined with Sulfotanshinone Sodium Injection in Treating Type II Optic Disc Vasculitis
}

\author{
Bingwen $\mathrm{Lu}^{1}$, Xingwei $\mathrm{Wu}^{2}$, Lei Zhang ${ }^{*}$ \\ ${ }^{1}$ Ophthalmology Department, Shanghai Seventh People's Hospital, Shanghai, China \\ ${ }^{2}$ Ophthalmology Department, Shanghai General Hospital, Shanghai, China \\ Email:^704487389@qq.com
}

How to cite this paper: Lu, B.W., Wu, X.W. and Zhang, L. (2016) Intravitreal Ranibizumab Combined with Sulfotanshinone Sodium Injection in Treating Type II Optic Disc Vasculitis. Case Reports in Clinical Medicine, 5, 494-499.

http://dx.doi.org/10.4236/crcm.2016.511062

Received: October 28, 2016

Accepted: November 19, 2016

Published: November 22, 2016

Copyright $\odot 2016$ by authors and Scientific Research Publishing Inc. This work is licensed under the Creative Commons Attribution International License (CC BY 4.0).

http://creativecommons.org/licenses/by/4.0/

(c) (i) Open Access

\begin{abstract}
Objectives: To present the effect of intravitreal ranibizumab (IVR) therapy combined with sulfotanshinone sodium (SS) injection in a patient suffering from type II optic disc vasculitis (ODV). Methods: A 26-year-old female patient was diagnosed with type II ODV with macular edema (ME). The information was obtained by complete medical and ophthalmic history taking and a detailed ophthalmic examination at the initial and follow-up visits. Functional and morphological outcomes at baseline, 1 week and 1 month following IVR+SS injections, are presented. Results: Bestcorrected visual acuity (BCVA) improved from 78 letters (ETDRS) at baseline to 90 letters at 1-week follow-up and maintained stable through 1-month follow-up. Central retinal thickness (CRT) measured by optical coherence tomography (OCT) decreased from $465 \mu \mathrm{m}$ at baseline to $240 \mu \mathrm{m}$ at 1-week follow-up and to $226 \mu \mathrm{m}$ at 1-month follow-up. Mean deviation (MD) measured by perimetry increased from $-5.17 \mathrm{~dB}$ to $-4.59 \mathrm{~dB}$ and to $-4.29 \mathrm{~dB}$, respectively. Fluorescein angiography (FFA) showed that the initial macular edema at baseline disappeared while the arm-retina circulation time (ART) was also greatly shortened when compared to the baseline. Electroretinogram (ERG) measured at 1-month follow-up demonstrated an overall improvement of the retinal function after the injection. No ocular or systemic side effects were detected. Conclusions: IVR+SS injection may lead to resolution of the associated $\mathrm{ME}$ and improve the retina morphologically as well as functionally. To our knowledge, this is the first case of a type II ODV benefiting from treatment with IVR+SS injection. The observed results warrant further investigation.
\end{abstract}

\section{Keywords}

Intravitreal Ranibizumab, Sulfotanshinone Sodium Injection, Type II Optic Disc Vasculitis 


\section{Introduction}

Optic disc vasculitis (ODV) is an uncommon, chronic and self-limiting disorder characterized by unilateral optic disc swelling and minimal visual symptoms, seen mostly in young adults with no gender predilection [1] [2]. Type II ODV is considered to result from phlebitis of the central retinal venous in the region of the optic nerve head or retro-laminar region and is usually misdiagnosed as central retinal venous occlusion (CRVO) because they share quite similar clinical pictures.

This 26-year-old female patient presented with obvious ME, exudation and hemorrhage with no big improvement after hormone therapy. Considering the similarities between these two diseases, we intended to apply our previous clinical experience in treating RVO patients on this patient.

Intravitreal injections of ranibizumab have been shown to be an effective treatment option for RVO patients [3] [4] [5]. Our previous studies have also demonstrated the therapeutic effect of SS injections in treating RVO patients [6] [7] [8]. This case study was designed to assess the efficacy and safety of IVR+SS infection in treating this type II ODV patient.

\section{Materials and Methods}

The information was obtained by complete medical and ophthalmic history taking and a detailed ophthalmic examination at the initial and follow-up visits.

Best-corrected visual acuity (BCVA) was 100 letters (ETDRS) OD and 78 letters OS at baseline. Anterior segment examination showed normal findings. Intraocular pressure (IOP) was $15 \mathrm{mmHg}$ bilaterally. Fundus examination showed diffuse optic disc edema with flame-shaped hemorrhages, macular exudation and hemorrhage as well as the twisting and dilation of the retinal veins in LE. Fundus examination showed normal findings in RE. Fluorescein angiography (FFA) demonstrated optic disc swelling, intraretinal hemorrhage and macular edema in LE. The arm-retina time (ART) was also obviously prolonged. The findings in RE were normal. Automated perimetry showed, in LE, a minimum defect linked with physiologic scotoma. Central retinal thickness (CRT) was measured by optical coherence tomography (OCT) as $465 \mu \mathrm{m}$. Electroretinogram (ERG) was also done to have an overall idea of the retinal function.

This 26-year-old female patient was treated with one IVR injection combined with SS injections (20 mg per day, five days consecutively in one week, 4 weeks). Written consent for the use of IVR therapy was obtained from the patient and a Ranibizuman (0.5 $\mathrm{mg}$ in $0.05 \mathrm{ml}$ ) (Lucentis, Genentech Inc.) was applied to the patient's left eye. SS injection $(5 \mathrm{mg} / \mathrm{ml}$ ) (batch number: H120921) manufactured by the First Biochemical Pharmaceutical Co. Ltd., Shanghai, China, is approved by State Food and Drug Administration of China. SS injection was given diluted at the point of treatment in $250 \mathrm{ml} 5 \%$ glucose injection for intravenous administration.

In addition to the treatment medicines, usages of any other traditional Chinese medicine or Western medicine were prohibited.

Except that FFA and ERG were done at one-month follow up, other examinations 
were repeated at one-week and one-month follow up.

\section{Results}

At 1 week following the IVR+SS injection, BCVA improved from 78 letters (ETDRS) at baseline to 90 letters at 1-week follow-up and maintained stable through 1-month follow-up. The CRT measured by OCT decreased from $465 \mu \mathrm{m}$ at baseline to $240 \mu \mathrm{m}$ at 1-week follow-up and to $226 \mu \mathrm{m}$ at 1-month follow-up. Mean deviation (MD) measured by perimetry increased from $-5.17 \mathrm{~dB}$ to $-4.59 \mathrm{~dB}$ and to $-4.29 \mathrm{~dB}$, respectively (Table 1). Retinal hemorrhage was reduced significantly demonstrated by the fundus examination and the macular edema had completely resolved as demonstrated by OCT through the 1-month follow-up (Figures 1-3). The arm-retina circulation time (ART) was also greatly shortened when compared to the baseline. ERG results showed an overall improvement of the retinal function as compared to the baseline (Table 2). IOP was measured unchanged at $15 \mathrm{mmHg}$ under given local therapy. No ocular or systemic side effects were detected.

Table 1. Clinical results of 1-month follow up.

\begin{tabular}{cccc}
\hline & BCVA (letters) & CRT $(\mu \mathrm{m})$ & MD $(\mathrm{dB})$ \\
\hline Initial & 78 & 465 & -5.17 \\
1 week & 90 & 240 & -4.59 \\
1 month & 90 & 226 & -4.29 \\
\hline
\end{tabular}

Table 2. ERG results of 1-month follow-up.

\begin{tabular}{cccccccccc}
\hline & Rod b & Max a & Max b & Max b/a & Osz total & Cone a & Cone b & Cone b/a & $\begin{array}{c}30 \mathrm{~Hz} \\
\text { Amp }\end{array}$ \\
\hline Initial & 59.6 & 206 & 439 & 2.1 & 162 & 49.5 & 153 & 3.1 & 46.4 \\
1 month & 199 & 210 & 516 & 2.5 & 178 & 50.3 & 165 & 3.3 & 48.8 \\
\hline
\end{tabular}
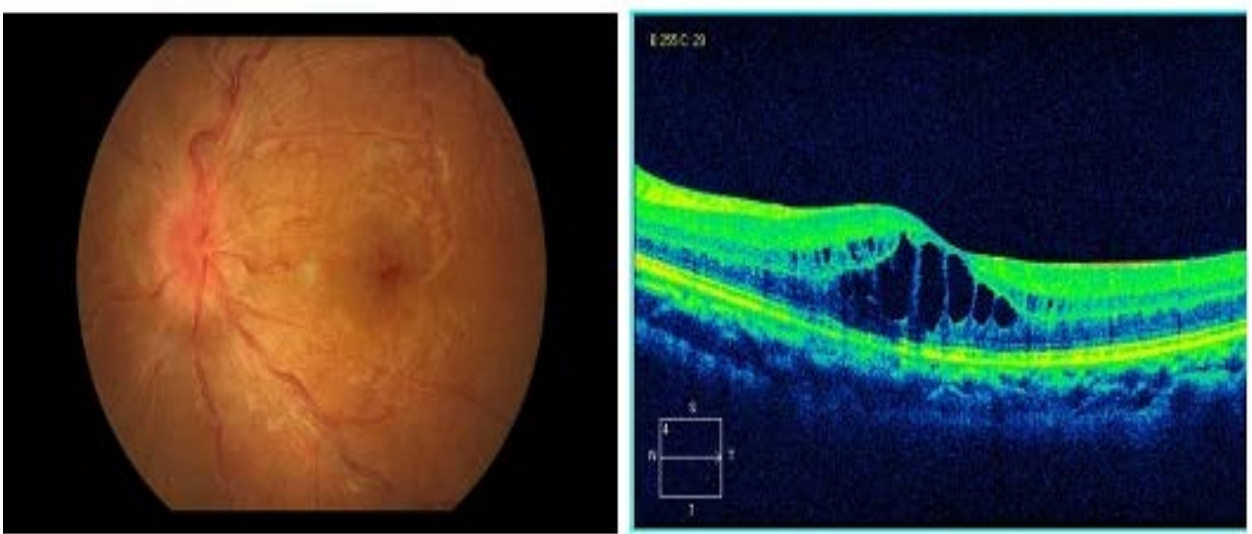

Figure 1. The fundus examination and OCT result at baseline. 

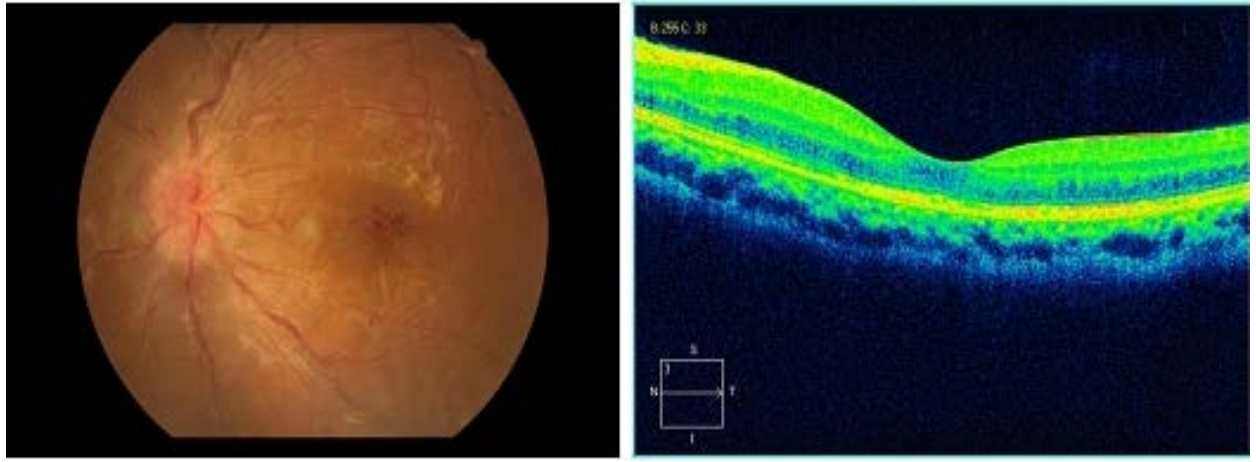

Figure 2. The fundus examination and OCT result at 1-week follow-up.
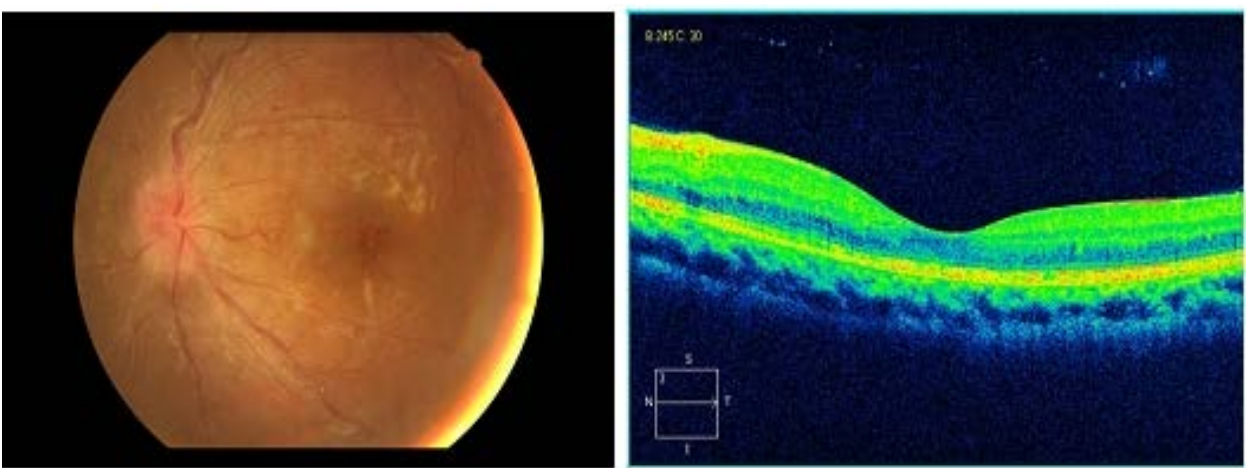

Figure 3. The fundus examination and OCT result at 1-month follow-up.

\section{Discussion}

Optic disc vasculitis (ODV) is an uncommon, chronic and self-limiting disorder characterized by unilateral optic disc swelling and minimal visual symptoms, seen mostly in young adults with no gender predilection [1]. Hayreh first described this clinical entity in 1972 [2]. Based on the clinical findings of optic disc and peripheral retinal hemorrhages, he subdivided his patients into two groups (type I and type II). Type I may result from mild non-specific vasculitis of the ciliary vessel in the prelaminar region. Type II may result from phlebitis of the central retinal venous in the region of the optic nerve head or retrolaminar region. Adequate systemic steroid therapy has a beneficial effect although the disease is usually self-limiting.

Type II ODV is usually misdiagnosed as central retinal venous occlusion (CRVO) for they share quite similar clinical pictures. However, the treatment and prognosis of them are different. Type II ODV is due to retinal phlebitis, so it exists mainly in young adults without hypertension, arteriosclerosis, diabetes mellitus or other systemic diseases with slightly impaired vision and good prognosis. Patients are usually sensitive to hormone therapies. Whereas, CRVO is due to arteriosclerosis, so it is mainly seen in elder adults with hypertension, arteriosclerosis, diabetes mellitus or other systemic diseases with evidently decreased eye sights and poor prognosis. Hormone therapies do not help.

This 26-year-old female patient, after hormone therapy for about two months, started to develop obvious macular edema, exudation and hemorrhage. Although her 
visual function was relatively good when she first came, the prognosis could be really bad if the ME progressed without any treatment. Current treatment options for patients suffering from type II ODV have limited visual outcomes. Considering the presence of severe ME, exudation and hemorrhage and considering the fact that this patient would not have benefited from either primary corticosteroids or further observation without treatment, we intended to combine the most up-to-date treatment therapy of RVO with SS injections based on our previous clinical experiences to try on this patient to avoid potentially poor visual outcome.

Current treatment options for RVO mainly include laser coagulation and anti-vascular endothelial growth factor (VEGF) therapy [3]. Direct laser coagulation has only limited beneficial effect on visual acuity, while anti-VEGF therapy has been shown to have great advantage in improving visual acuity. Inhibitors of VEGF can prevent the formation of abnormal blood vessels and counteract VEGF-induced vascular permeability. Within the eye, VEGF-related vascular pathologies like neovascular age-related macular degeneration (nvAMD) can be treated with ranibizumab (Lucentis, registered for the treatment of nvAMD), an antibody-fragment directed against VEGF, or with bevacizumab (Avastin), an anti-VEGF IgG approved for advanced colon cancer and used off-label in ophthalmology. The intravitreal injection of VEGF-inhibitors, such as ranibizuman (IVR) or bevacizumab (IVB), is a new treatment modality for macular edema due to various underlying diseases. Ranibizumab (Lucentis) has shown to reduce vascular permeability in choroidal nwovasculariation due to nvAMD as well as in pathological capillaries in diabetic macular edema and in macular edema due to retinal vein occlusion. The results from the CRUISE and BRAVO trials of ranibizumab in the treatment of RVO-associated macular edema suggested efficacy and safety of ranibizumab for this indication [4] [5].

Danshen is considered to promote blood circulation for removing blood stasis and improve microcirculation. It has been widely used to treat cardiovascular diseases for more than 2000 years in China. TSA is an herbal monomer with a clear chemical structure, isolated from Danshen. Previous animal studies have found that compound Danshen dripping pills could be a promising method for the treatment of ischemic retinal disorders through reducing oxidative stress [6]. Our previous clinical study has demonstrated that SS injection was an effective and safe modality for treating RVO patients [7]. We also have shown that IVR injection and SS injection together could effectively improve the therapeutic effect in RVO patients with ME [8]. Therefore, we treated this patient with one intravitreal injection of ranibizumab (IVR) combined with SS injections to see the therapeutic effect on her ME and her entire retina function.

We treated this patient with one IVR injection combined with SS injections to see its effect on her ME and her entire retina function. The results demonstrated the efficacy of IVR+SS therapy in reducing ME. The ERG and perimetry results also showed that this treatment could significantly improve this patient's retinal function and visual function.

To our knowledge, this is the first patient who was treated with IVR+SS injection 
therapy for type II ODV. The result of this case showed rapid decrease in CRT and visual improvement with one-month follow up so far. Considering the strength of evidence, a long time follow-up need to be conducted in this investigation to assess the effect of this treatment method, and more rigorously designed trials are required for assessing the clinical application of this combined therapy to treat type II ODV patients.

\section{Conclusion}

In summary, IVR injection and SS injection together could effectively improve the visual acuity and rapidly alleviate the edema in this type II ODV patient.

\section{References}

[1] Erdurman, F.C., Durukan, A.H., Mumcuoğlu, T. and Hürmeriç, V. (2009) Intravitreal Bevacizumab Treatment of Macular Edema due to Optic Disc Vasculitis. Ocular Immunology and Inflammation, 17, 56-58. https:/doi.org/10.1080/09273940802491868

[2] Oh, K.T., Oh, D.M. and Hayreh, S.S. (2000) Optic Disc Vasculitis. Graefe's Archive for Clinical and Experimental Ophthalmology, 238, 647-658. https:/doi.org/10.1007/s004170000157

[3] Girmens, J.F., Glacet-Bernard, A., Kodjikian, L., Nghiêm-Buffet, S., Massé, H., Fourmaux, E., Wolff, B., Roquet, W., Gaucher, D., Baillif, S. and Tadayoni, R. (2015) Management of Macular Edema Secondary to Retinal Vein Occlusion. Journal Français D'Ophtalmologie, 38, 253-263. https:/doi.org/10.1016/j.jfo.2014.10.003

[4] Campochiaro, P., Heier, J., Feiner, L., et al. (2010) Ranibizumab for Macular Edema Following Branch Retinal Vein Occlusion; Six-Month Primary End Point Results of a Phase III Study. Ophthalmology, 117, 1102-1112. https:/doi.org/10.1016/j.ophtha.2010.02.021

[5] Brown, D., Campochiaro, P., Singh, R., et al. (2010) Ranibizumab for Macular Edema Following Central Retinal Vein Occlusion: Six-Month Primary End Point Results of a Phase III Study. Ophthalmology, 117, 1124-1133. https:/doi.org/10.1016/j.ophtha.2010.02.022

[6] Lu, B.W. and Wu, X.W. (2015) The Protective Effect of Compound Danshen Dripping Pills on Oxidative Stress after Retinal Ischemia/Reperfusion Injury in Rats. Chinese Medicine, 6, 90-96. https:/doi.org/10.4236/cm.2015.62010

[7] Lu, B.W. and Wu, X.W. (2015) Clinical Study of Sulfotanshinone Sodium Injection in Treating Non-Ischemic Retinal Vein Occlusion. Chinese Medicine, 6, 83-89. https:/doi.org/10.4236/cm.2015.62009

[8] Lu, B.W. and Wu, X.W. (2015) Retinal Functional Changes Measured by Microperimetry after Intravitreal Ranibizumab Injection and Sulfotanshinone Sodium Injection for Macular Edema Secondary to Retinal Vein Occlusion. Chinese Medicine, 6, 181-186.

https:/doi.org/10.4236/cm.2015.63020 
Submit or recommend next manuscript to SCIRP and we will provide best service for you:

Accepting pre-submission inquiries through Email, Facebook, LinkedIn, Twitter, etc. A wide selection of journals (inclusive of 9 subjects, more than 200 journals)

Providing 24-hour high-quality service

User-friendly online submission system

Fair and swift peer-review system

Efficient typesetting and proofreading procedure

Display of the result of downloads and visits, as well as the number of cited articles

Maximum dissemination of your research work

Submit your manuscript at: http://papersubmission.scirp.org/

Or contact crcm@scirp.org 